\title{
A New Chapter for Cognitive, Affective \& Behavioral Neuroscience
}

\author{
Diego A. Pizzagalli ${ }^{1}$
}

Accepted: 13 January 2021 / Published online: 16 February 2021

(C) The Psychonomic Society, Inc. 2021

On January 1, 2021, I assumed the Editor-In-Chief role for Cognitive, Affective \& Behavioral Neuroscience (CABN). I feel humbled and excited to have an opportunity to steer and shape a journal that has had such a large impact across different domains of neuroscience for decades. My talented Associate Editors and I have inherited a journal that has been thoughtfully guided by Dr. Marie Banich (and before her, Dr. Deanna Barch), who has done an outstanding job as Editor. The goal of the new editorial team will be to further grow and strengthen the journal; we hope to achieve this goal by leveraging CABN's unique place in the field and by implementing some key changes.

Importantly, CABN's mission will remain the same-it will continue to serve as "the leading vehicle for strongly psychologically motivated studies of brain-behavior relationships, through the presentation of papers that integrate psychological theory and the conduct and interpretation of the neuroscientific data" (https://www.springer.com/journal/ 13415). True to its name, CABN will continue to cover a broad range of topics, including perception, attention, memory, language, problem solving, reasoning, decisionmaking, affect, and motivation. Moreover, we welcome research conducted with heathy participants, neuropsychiatric or neurological patients, or nonhuman animals. Clearly, CABN has a broad reach; nevertheless, the critical common denominator will be a strong focus on "psychologically motivated studies of brain-behavior relationships."

To be more specific about the manuscripts that will versus will not be within the scope of the journal:

- A manuscript focused solely on behavioral or computational data that are not conceptualized within a

Diego A. Pizzagalli

dap@mclean.harvard.edu

1 McLean Hospital, Harvard Medical School, 115 Mill Street, Belmont, MA 02478, USA neurobiological framework will likely not be sent out for review. To be explicit: CABN will publish manuscripts featuring only behavioral or computational data, but only if they are clearly embedded within a neuroscientific context or theory. For instance, a study that assessed patients with Parkinson's disease both on and off medication to elucidate the role of dopamine on cognition would be welcome, so would studies that used a drift diffusion model to study evidence accumulation in healthy or disease states, or that focused on variables (e.g., pupil dilation) that have been clearly linked to precise neurobiological underpinnings in prior work.

- A manuscript focused exclusively on the clinical aspects of a neuropsychiatric condition (e.g., depression, frontotemporal dementia) will likely be judged to lie outside of CABN's focus. Conversely, manuscripts that link such conditions to brain function (e.g., via lesion analysis), or that involve testing a specific neuroscientific theory, will be of great interest to CABN.

- A review summarizing biomarkers associated with a given disorder (e.g., schizophrenia) that is not psychologically motivated — or that does not leverage brain-behavior relationships to elucidate psychological processes disrupted in the disorder-will be deemed outside CABN's focus. By contrast, a review that explicitly makes such links will almost certainly be considered.

- A manuscript solely focused on how a drug affects neural function would lie outside CABN's scope. CABN will, however, eagerly accept submissions from preclinical studies, particularly those using cutting-edge techniques to mechanistically manipulate neural systems (e.g., optogenetics, designer receptor exclusively activated by designer drugs [DREADDs]), as long as they carefully evaluate the behavioral consequences of those manipulations.

- CABN also remains strongly interested in computational modeling, because this approach quantifies psychological constructs and the relationships among them, and often provides a formal way to compare findings across species. 
In sum, $\mathrm{CABN}$ will continue what has been thoughtfully accomplished under the two prior editorships (Banich \& Barch)_namely, publishing studies that test a psychological model or hypothesis with methods that rigorously link brain and behavior.

In support of CABN's continual improvement, we have also implemented some changes. First, we have increased the diversity of our consulting editors, where "diversity" includes area of expertise, geographical location, faculty rank, gender, and - critically - underrepresented minorities. Fiftyfive percent of the consulting editors (23 of 42) are new to CABN; they are joining a group of outstanding scientists from around the globe who we are grateful to have onboard. We will rely on our strong team of consulting editors to provide thoughtful, constructive, and prompt reviews that will help authors publish impactful work.

Second, and along similar lines, we have increased the range of expertise among the Associate Editors. In this regard, I am delighted to write that I have enlisted five outstanding scientists with substantial expertise as Associate Editors: Drs. Senne Braem (Ghent University, Belgium; expertise in cognition and reward); Nancy A. Dennis (Penn State University, USA; expertise in memory); Stan B. Floresco (University of British Columbia, Canada; preclinical expertise in learning and cognition); Carly J. Leonard (University of Colorado Denver, USA; expertise in attention and vision); and Daniela Schiller (Icahn School of Medicine at Mount Sinai, USA; expertise in learning, emotion, decision-making, and social cognition).

Third, we will shorten the review process: reviewers will be asked to return their evaluations within 3 weeks, and we will aim to have a first decision within 1 month. We believe that efficient, fair, and constructive reviews will serve authors and CABN well. For my part as the Editor-In-Chief, I will facilitate this process by personally reviewing every new submission to evaluate whether it should be sent out for review or not, either because it falls outside the scope of CABN or is unlikely to compete successfully for the limited space in the journal.

Fourth, we will continuously evaluate metrics linked to diversity and inclusion, broadly construed. We are committed to doing our part to ensure that representation on our board is aligned with the percentage of women and underrepresented minorities within neuroscience, and we will be on the lookout for any biases in our invitations to review or publish manuscripts.

To conclude, there have been so many positive developments recently - including new imaging techniques, increased focus on rigor and reproducibility, more emphasis on modeling, and the critical focus on inclusion and diversity - that neuroscience is in an exciting new phase. The field is ideally poised to offer new insights into behavior, in both health and disease, and to help refine psychological theories that can explain that behavior. We want CABN to be a leading outlet for your work on these topics, and we look forward to receiving your submissions. For any feedback or suggestions you may have, feel free to contact me at dap@mclean.harvard.edu.

\section{Disclosures}

Over the past 3 years, Dr. Pizzagalli has received consulting fees from BlackThorn Therapeutics, Boehringer Ingelheim, Compass Pathway, Concert Pharmaceuticals, Engrail Therapeutics, Otsuka Pharmaceuticals, and Takeda Pharmaceuticals; one honorarium from Alkermes, and research funding from the National Institute of Mental Health, Dana Foundation, Brain and Behavior Research Foundation, Millennium Pharmaceuticals. In addition, he has received stock options from BlackThorn Therapeutics. No funding from these entities was used to support the current work, and all views expressed are solely those of the author.

Publisher's note Springer Nature remains neutral with regard to jurisdictional claims in published maps and institutional affiliations. 